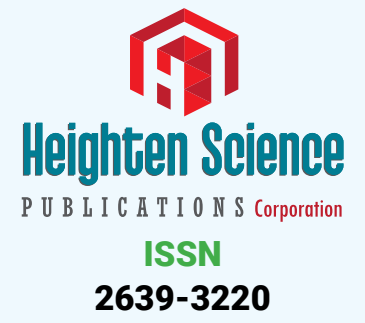

*Address for Correspondence: Alessandro Giustini, Scientific Director, San Pancrazio Rehabilitation Hospital, Chair of Robotic and New Technologies Scientific Committee, European Society of Physical and Rehabilitation Medicine, Arco, Trento, Italy, Tel: +39 3473807592; Email: alessandro.giustini@ntc.it

Submitted: 22 October 2018

Approved: 27 November 2018

Published: 28 November 2018

Copyright: (c) 2018 Giustini A. This is an open access article distributed under the Creative Commons Attribution License, which permits unrestricted use, distribution, and reproduction in any medium, provided the original work is properly cited

A) Check for updates
Review Article

\section{Neuroscience, Rehabilitation and New Technologies: Perspectives and critical points for a synergistic development}

\author{
Alessandro Giustini* \\ Scientific Director, San Pancrazio Rehabilitation Hospital, Arco, Trento, Italy
}

\section{Forewords}

The growth of Rehabilitation, in all of its field and mainly in neuro-rehabilitation applications and settings, is showing increasingly strong interaction with the growth of technology and its innovative applications.

Nevertheless, is should be stressed that the use of machinery has always been a fundamental mainstay of Rehabilitation practices facing the whole person's aspects and involving the whole physical world around the disabled people as it is: as it was in the past with physical exercises, physical modalities, and in many other trainings and activities that employed physical and technological means as Aids, Prostheses and Orthotics.

So many new possibilities offered day by day by technologies actually support a continuous development for traditional rehabilitation tools, a continuous enlargement for their applications toward the better recovery of functioning and health for any subject with disability.

Today, on the other hand, the peculiarity of new technological equipments and methodologies for evaluation, but mainly for treatments, is interacting actively and profoundly with rehabilitation practices very often in some neurological conditions, very often subverting many previously shared theoretical, clinical and management paradigms.

So many different applications (not only new ones) of technologies are fundamental in therapeutic interventions and in Activity /Functioning recovery towards better individual health condition.

What is robotic and what assistive (new) technology?

A large difficulty arises from a classification, not well defined at the moment of the types and categories of these devices and apparatus regarding the so many differences in characteristics, utilization, aims etc.

There is until now no complete consensus on which machines can be qualified as Robots but there is general agreement among experts that robots tend to do some or all of the following: move around, operate a mechanical limb, sense and manipulate their environment, and exhibit intelligent behavior, especially behavior which mimics humans or other animals.

It can summarized as follows: Robots have actuators and sensors, the action they are performing is based on the sensed status or environment and there is an intelligent reaction to this status or environment. Without the intelligence it is "only" an automat. 
Rehabilitation robotics is a special branch of robotics, which focuses on machines that can be used to help people recover from severe physical trauma [1]. The born of this speciality is unclear but the first clinical robot application can be in the 1960s with Powered Human Exoskeleton Devices. Its context has varied between tools for delivering repetitive training; to tools to influence relearning lost motor skills in an engaging and therapeutic context. Numerous robotic devices for recovery lower or upper limb or hand function with various levels of complexity and functionality have been developed over the last 10 years. In clinical practice a robotic device for rehabilitation was define as any technology that has the ability to assist the patient's limb movement for therapeutic exercises and able to support the therapist during administration of programmable and customized rehabilitation programs, composed by mechanical structure with actuators and energy supply.

Two main approaches have been used to design upper and lower limb rehabilitation devices: end-effector and exoskeleton with or without a haptic system control.

On the other hand Assistive Technology is defined as "any item, piece of equipment, or product system whether acquired commercially off the shelf, modified or customized, that is used to increase, maintain, or improve functional capabilities of individuals with disabilities. It is a broad range of devices, services, strategies, and practices that are conceived and applied to ameliorate the problems faced by individuals who have disabilities."

AT devices are tools for enhancing the independent functioning of people who have impairments or disabilities [2,3]. They range from low-tech aids, such as built-up handles on eating utensils, to high tech devices such as computerised communication systems, alternative access systems or powered wheelchairs. The ultimate objective of AT is to contribute to the effective enhancement of the lives of people with disabilities and elderly people by helping to overcome and solve their functional problems, reducing dependence on others and contributing to the integration into their families and society $[4,5]$.

It is not so easy to define exactly the kind of some devices: for example a wearable exoskeleton (whit sensors and actuators, intelligent reactions for the training and for the independent standing /walking) belongs to robot or only to new assistive technology?

Only a point is easy to understand: as it is useful to enrich and motivate the training and to upgrade results!

These definitions have several important elements. Both emphasise the functional capabilities of individuals with disabilities as a result of the successful use of these different devices and take a strong perspective on the outcomes in terms of quality of life. It underscores the importance of assessing and supporting the unique needs of each individual and the context in which they will be applying the devices.

Nevertheless, it is growing other different kinds (Virtual Reality and Telerehabilitation) of new applications of technology in rehabilitation: this development is a natural consequence of the rapid expansion of electronics and communication technology, providing new possibilities for independent living for individuals with neurological and other disabilities.

It is really not so easy to combine these devices in relation to the previous 2 definitions: the only key-point could be "New (advanced) technologies "and could be sufficient.

The application of telerehabilitation as an adjunct to traditional clinical service may offer major benefits, particularly in terms of improved communication and access to health care over distance. Improved communication allows information sharing or even 
medical data exchange between patients, family, carers, clinicians, and researchers. It also may be a stimulating factor for extending the dialogue between patients and carers or clinicians and as such may be a potential source of augmented feedback. Access to health care over distance offers people living in remote areas the possibility to access to health care services or allows access to health care in specialised facilities located at longer distance from the place where the patient is living. Other potential advantages of access to health care over distance are the possibility of early diagnosis and start of therapy in acute situations, improving continuity of (specialised) care with possibilities for shortening length of stay in hospital or specialised rehabilitation centre, continuous monitoring of people at risk, and therefore reducing travelling costs and time between home and health service centre [6].

Telerehabilitation appears to be often applied as an adjunct to virtual reality tools when these are used in the home environment. Virtual reality techniques and high speed networks create an environment allowing clinicians and technical staff at different sites to interact with 3-dimensional visualisation of patient-specific data [7]. Firstly some years ago was reported the development of a PC-based orthopaedic rehabilitation for use at home, while allowing remote monitoring through videoconferencing from the clinic, and also the application of a system for rehabilitation of hand, elbow, knee and ankle impairments on the basis of virtual reality, haptic interfaces and networked PC's to provide exercises while being monitored remotely by therapists. A library of virtual rehabilitation routines was developed, consisting of three physical therapy exercises (Digikey, ball, and power putty) and two functional rehabilitation exercises (peg board and ball game).

In recent years many different systems was developed for training in virtual rehabilitation and often telerehabilitation (cognitive and speech problems, balance, upper and lower limbs ...) and their diffusion is well known [8]. Not necessary a diffusion as "Home Rehabilitation" but mainly as distance-rehabilitation, even if remaining into the Rehabilitation Centre to reduce costs for personnels and implement time for training.

To be able to face to these new aspects, showing how these are parts (really new but completely inner to the scientific and practical role of rehabilitation specialists) belongs to the our Discipline evolution, showing also if and how many other professionals and different technicians can cooperate under the guidance of Rehabilitation Specialist in the team, with the common aim to enrich the scientific results and outcomes for Peoples in treatment, showing how any evaluation for research and for certifications regarding these new technologies and their clinical applications belongs to Rehabilitation :all that is today fundamental to support and expand this activity in clinical and research fields [9-12].

In Europe we are actually trying to define a common vision all around these problems, recognizing and understanding the main points regarding the competences and activities of PRM Doctors being the main rehabilitation medical specialist in any health condition; the tools are scientific aspects (really litter at now) but mainly professional, clinical and management experiences and opinions about how rehabilitation processes and facilities must be guided, about how education and research must be addressed, about how relationship with other professionals must be utilized.

One of the main key point is surely regarding the better involvement of knowledge and indications from neuroscience, not only regarding neuro-rehabilitation clinical fields, procedures and interventions, but regarding any robotic application in relation to the fundamental active cognitive relationship between device and person with disability.

Another key point is surely the better involvement of knowledge (and activity directly in clinical team and facilities) of technicians (bioengineers, ICT professional) creating a sort of common language, a common vision about common goals together health professionals. 
Some critical remarks: Key-problems for rehabilitation.

This field takes concrete form, in particular, with projects and experiences for the use of innovative equipment and technological systems as supports, interaction or instruments for the realisation of various types of treatment, for different purposes.

This interaction discloses great positive aspects, but also strong critical points due to the objective difficulty in correlating the actual needs of rehabilitation practices with appropriate responses on the part of technological research, as well as with verification of the efficacy and efficiency of these innovations.

We all openly can see the growth and quick diffusion everywhere of these offers in a concrete "market" (involving science, hope and illusion) very often subverting rules of care for health: many Companies are strongly promoting these instruments more and more in our Countries, scientific literature shares interesting experiences in Europe and all over the world, commercial advertising is strongly present near (TV, Newspapers etc.) people, families and disabled people Associations [13].

One of the most important criticisms in this "market" is that disabled people (his family and community) needs to can choose cares not only referring to the scientific/ medical/traditional basis and standards but mainly referring to the "client satisfaction" that means " disabled satisfaction" regarding individual health, participation and quality of life.

Goals for Health Services are changing in all of our countries under the power of free choices done by people and patients : not only to reach recovery but more often to reach a time of well-being, having the possibility, longer as possible, to maintain the best level of Participation and Authonomy.

Rehabilitation can be the key-word, and the key-role!

So the strongest tool for us is to apply ICF in all its value: to guide information to patients, researches, measures, indications, results in patients (persons) life, evidences, efficacy/effectiveness (also regarding finances and fees).

ICF must be the cornerstone for our methodology in any activity, and firstly the cornerstone for our education re-directing and strengthening all our knowledge and competences.

All these arguments involve the whole rehabilitation field, but surely robotic and new-technologies have a special value in this development.

For example:

- Are totally inside and promote these changes,

- Can offer more and more changes,

- Bring these new possibilities to care directly to the whole person (physical, cognitive, relational, environmental aspects) underlining his authonomous action.

But unfortunately, as in any relevant transformation, the change can assume a positive or negative direction: there are some critical aspects that our ethical, scientifically and professional duties must face.

Also in the awareness as needed by disabled people rights, and by sustainability of Health Services.

\section{A) Lack of complete clinical instructions before the "marked" diffusion}

The first and big critical point is often (and for many of the equipment on the 
market) that there are no specific clinical elements of rehabilitation evidence to support the proposals that companies introduce [14]. Often there is only basic but simple kinesiological, static-mechanical or neuro-functional evidences; in other cases there are only cognitive, behavioural, sensorial and relational elements, which are however entirely simple and elementary and are definitely not correlated to overall processes of understanding, learning and functional acquisition for the recovery in functioning and participation.

Also the References, actually increasing more and more, don't yet offer evidences suitable for the many and different applications.

In this sense, there is a great need for overall rehabilitative verifications on existing equipment, as well as for rehabilitative research to better orient companies' technological applications in future (to modify the existing or to create new ones .

Moreover, some great positive perspectives (to be developed) also emerge:

First of all, the possibility to have new tools to study and clarify modalities of therapeutic interventions, of learning recovery and evidences in our clinical activities;

The possibility to guarantee great homogeneity and measurability of treatments, as well as of the relative effects and functional results;

The possibility, therefore, to realise wide-ranging and significant clinical studies, to bring out evidence of a biomechanical and functional nature and on individual performance.

\section{B) Needs for new management and organization}

Other critical elements in this phase turn up in the field of management and organisation (in the facilities, in the individual rehabilitation project, in the medical prescriptions and follow-up) to apply these instrument, but also to obtain the resources that this development requires.

As a matter of fact very often these new modalities for treatment have not a specific recognition, quite if it is the same of the other "traditional" way: one of the most important cause is the point A, but also the financial crisis in Health Services in any Country is important too.

Therefore arises limitations and differences among Rehabilitation Centres that can equip (or not) themselves with this innovative machinery, giving rise to doubts as to disparities in treatment among the many types of persons with disabilities to submit (productively?) to treatments.

Moreover, some great positive aspects (to be developed) also emerge:

The possibility to implement tele-rehabilitation on a large scale, in its different forms, understood as an appropriate form of continuity and effectiveness of treatments, integrating them with the environment, individual and motivational lifestyles of Persons who are deeply and actively involved.

Last but not least, a concrete possibility to multiply care in relation to the multiplication of demand on the part of persons with disabilities, without excessive conflict with the costs that the use of only personnel would produce.

\section{C) New Education}

Additional critical elements that emerge is the objective need to modify the contents of training for Rehabilitation Team personnel, for the appropriate and widespread use of technological equipment in rehabilitative treatment, as well as the organisation of various activities in the different temporal phases of treatment [15]. 
Also the relationship between professionals and patients is modified with respect to the substantial aspects of accepting patients for care and therapy and conducting the treatment between the patient and operational personnel. The change for doctors is mainly in research and define new clinical paradigms, protocols and guidelines, adding to the previous knowledge.

The question now arises as to whether this equipment can, perhaps partially regarding some traditional activities, replace specific categories of operational personnel needed to cooperate with Medical Specialists (Physiotherapists, Speech and Language Therapists, Occupational Therapists); or perhaps substitute rehabilitative settings where therapeutic programmes are traditionally carried out (Tele-rehabilitation).

In analysing relations between operational personnel and equipment, the theme of "difficulty" in performing work is also posed, which tends to show up with Physiotherapists (etc.) and which is also one of the elements of adaptation for patients (for their hardships), in their progress in performing exercises [16].

The machinery, on the other hand, is not subject to these adaptations and we should imagine further "intelligent" evolution to enable it to adapt productively to reactions of persons undergoing therapy.

Another aspect (only a brief note but the point has a wide scientific contents from the patients and for the professional too) is the "fatigue" in the training and in the single exercise, which modify the repetitions, the strength, the characters etc. Fatigue can underline or involve aspects regarding attention (not only by patient), participation, motivation.

Obviously the machine do not have fatigue! But could be necessary to adapt the activity programmes in the machinery to avoid problems connected with risks of fatigue for the patients during the training.

So the problem is precisely the specific (new) competence for operational personnel, who can survey and guarantee the exactness of these therapeutic procedures. Surely it is quite new regarding the traditional previous role for professionals: realizing the treatment directly "by their hands".

It needs a quite different new education for these professionals, a quite new role in the global management of the treatment programmes, times, measurements and responsibilities.

Neuroscience has a specific key role to be enlarged in educational programmes for medical specialists (PRM mainly involved in rehabilitation activities, often together Neurologists, Gerontologists and others) and for all other professionals, technicians too as bioengineers.

Regarding this matter in Europe there are growing some interesting innovative project to develop education integrating different professional profiles, and in the same time upgrading rehabilitation in the health system.

TechReh ERASMUS+ Project can be an interesting recent example, financed by the European Union in Uzbekistan. The main objective of TechReh project is to define a learning environment to deliver more opportunities to access new competences related to the rehabilitation activities and jobs. These new competences refer, in particular, to the use of advanced ICT solutions for the rehabilitation [17]. TechReh would fulfill the goal of optimization of the healthcare organizations network stated in the Welfare Improvement Strategy of the Republic of Uzbekistan.

At the beginning of the program a state of art was realized and, then, needs-analysis was accomplished, followed by the development of some specific objectives of TechReh: 
1) Identification of technological needs for rehabilitation in Uzbekistan, their experiences in the field are essential to define the background and how EU experiences, polices, best practices can improve the current situation;

2) Deploying of a Formative Programme in the field of Medical Rehabilitation on the use of advanced technological solutions in rehabilitation to train Doctors and Medical Operators on innovative devices and interventions: In the same time to upgrade formative programmes for technicians to be able to well cooperate together medical and health professional in research and clinical activities.

3) Setup of Offices for Cooperation and Dissemination of Technology in Rehabilitation (OCDTRs) in order to consolidate the technology adoption and development in rehabilitation fields;

4) Setup of an ICT platform for the dissemination of didactic materials, contents, and project results and developments.

Actually this Project is going to finish in 2019 and the changes in Education and Health National systems in Uzbekistan are yet visible and well working.

\section{D) Care relationship}

Actually, due to the effects of this equipment, the position and attitude of patients towards their programme of recovery and towards the proactive behaviour they must endeavour to implement in its realisation is very often substantially modified as well.

The primary need is therefore confirmed of global and individual care for the Person in the Individual Rehabilitation Project, in order to bring every intervention to a therapeutic and evaluation synthesis. This situation, much more complex, request a solid role for responsibility in the hands of rehabilitation medical responsible obviously regarding the prescriptions for technological treatments, evaluations of results, coordination with other interventions in the recovery program [18-20].

The equipment is sometimes included as a "support and multiplier" to extend activities and treatments at every level, maintaining a relation with the "traditional" care program regarding the different clinical situations. But the machine on the contrary for example can show results on time, can create a sort of "positive game "for the patients, and directly communicating to the patients, in such a way excluding the professionals.

Other times the equipment is introduced as an entirely new potential (which would otherwise not be possible) to perform rehabilitative training that must in any case be included in organic care programmes, under the guidance of the physician and with the intervention of adequately prepared operators.

So immediately, in both cases, the equipment creates a new situation in which the information, the involvement and communications about results must be up-grade toward patients, family and care-givers: it is better also regarding to the active role of the Person in care as necessary.

\section{Brief Conclusion}

Obviously this last point together all the previous ones, is only a part in a global matter and the solutions must be global finding a solution for the two main key problems underlined previously: better and deeper connections between rehabilitation and neurosciences in one hand, and bioengineer in another hand both in research and in clinical activities.

The goal must be to have large knowledge regarding applications, effects and indications, based on evidences connecting technical, biological, functional and psychosocial aspects of these treatments and their outcome for disabled people.

These are the hoped Great Perspectives underlined in the Title. 


\section{References}

1. Delarque A, Franchignoni F, Giustini A, Lankhorst G. European Physical and Rehabilitation Medicine: 3 years after White Book. J Rehabil Med. 2010; 42: 1-3. Ref.: https://goo.gl/zyzGnv

2. Giustini A. "Certainties and prospects in PRM". EuraMedicoPhys. 2005; 41: 215-219.

3. Giustini A. From acute intervention to domiciliary and social integration: research to build CommunityBased Rehabilitation (CBR) on effectiveness". International Journal of Rehabilitation Research. 2004; 27 suppl 1: 44-45.

4. Sandrini G, Binder H, Hömberg V, Saltuari L, Tarkka I, et al. European core curriculum in neurorehabilitation. Funct Neurol 2017; 32: 63-68. Ref.: https://goo.gl/ZvaFMM

5. Giustini A, Varela E, Franceschini M, Votava J, Zampolini M, et al. UEMS -Position Paper. New Technologies designed to improve Functioning. Eur J Phys Rehabil Med. 2014; 50: 579-583. Ref.: https://goo.gl/ZVTkqP

6. Lo AC, Guarino PD, Richards LG, Haselkorn JK, Wittemberg GF, et al. Robot-assisted therapy for long-term upper limb impairment after stroke. N Engl J Med. 2010; 362: 1772-1783. Ref.: https://goo.gl/YpY4RS

7. Holden MK. Virtual environments for motor rehabilitation: review. 26. Cyberpsychol behave. 2005; 8: 187-211. Ref.: https://goo.gl/AnHicA

8. Huijgen BC, Vollenbroek-Hutten MM, Zampolini M, Opisso E, Bernabeu M, et al. Feasibility of home-based telerehabilitation system compared to usual care: arm/hand function in patients with stroke, traumatic brain injury and multiple sclerosis. J Telemed Telecare. 2008; 14: 5 -20. Ref.: https://goo.gl/cdoK4C

9. Man DW, Soong WY, Tam SF, Hui-Chan CW. A randomized clinical trial study on the effectiveness of a tele-analogy-based problem-solving programme for people with acquired brain injury (ABI). NeuroRehabilitation. 2006; 21: 205-217. Ref.: https://goo.gl/1caGaM

10. Mehrholz J, Thomas S, Werner C, Kugler J, Pohl M. et al. Electromechanical-assisted training for walking after stroke. Cochrane Database of Syst Rev. 2007: CD006185. Ref.: https://goo.gl/v7Bhw1

11. Mehrholz J, Platz T, Kugler J, Pohl M. Electromechanical and robot-assisted arm training for improving arm function and activities of daily living after stroke. Cochrane Database of Syst Rev. 2008; CD006876. Ref.: https://goo.gl/XWNwVL

12. Piron $L$, Tonin $P$, Piccione $F$, laia $V$, Trivello $E$, et al. Virtual 17. Environment training therapy for arm motor rehabilitation. Presence 2005; 6: 732-740. Ref.: https://goo.gl/5YTgt9

13. Reinkensmeyer DJ, Pang CT, Nessler JA, Painter CC. Web-based telerehabilitation for the upper extremity after stroke. IEEE Trans Neural Syst Rehabil Eng. 2002; 10: 102-108. Ref.: https://goo.gl/ZLsBjs

14. Sale $P$, Franceschini $M$, Waldner A, Hesse S. Use of the Robot Assisted Gait Therapy in rehabilitation of patients with stroke and spinal cord injury. Eur J Phys Rehabil Med. 2012; 48: 111-121. Ref.: https://goo.gl/jfV3W4

15. Stucki G, Grimby G. Applying the ICF in Medicine. J Rehabil Med. 2004; (44 Suppl): 5-6. Ref.: https://goo.gl/5uFLiS

16. United Nations. Convention on the rights of persons with disabilities (resolution 61/106). New York, NY: United Nations; 2007. Ref.: https://goo.gl/NW88wr

17. Ustun B, Chatterji S, Kostanjsek N. Comments from WHO for the Journal of Rehabilitation Medicine Special Supplement on ICF Core Sets. Journal of Rehabilitation Medicine. 2004; (44 Suppl): 7-8. Ref.: https://goo.gl/euTkEW

18. Von Groote PM, Giustini A, Bickenbach JE. Analysis and Implementation of a WHO Health Report. Methodological Concepts and Strategies. Am J Phys Med Rehabil. 2014; 93 (1 Suppl 1): S12-26. Ref.: https://goo.gl/eWx9fP

19. Wade DT. Community rehabilitation, or rehabilitation in the community? Disabil Rehabil. $2003 ; 25$ : 875-881. Ref.: https://goo.gl/HKyKtw

20. World Health Organization. World Report on Disability. Geneva: World Health Organization; 2011. Ref.: https://goo.gl/GbDhoS 